\title{
Can Quantitative CT Texture Analysis be used to Differentiate Subtypes of Renal
}

\section{Cell Carcinoma?}

\section{Introduction}

Renal cell carcinoma (RCC) is a common malignancy with an increasing mortality rate $^{1,2}$. It is a heterogeneous disease with different histological subtypes. Clear cell, papillary and chromophobe RCC are the most common subtypes ${ }^{2}$. The histological classification of RCC is important as clear cell RCC (ccRCC) generally has a worse prognosis compared to other subtypes ${ }^{3,4}$. As the available targeted agents of metastatic RCC (mRCC) mainly relate to $\mathrm{ccRCC}^{5-7}$, the optimal treatment for mRCC with nonclear cell histology is still under investigation. A tumor biopsy is indicated to provide histological information before commencing systemic treatment, but its invasive nature and unsatisfactory accuracy have limited its clinical application in certain patients ${ }^{8}$. It would be helpful if there is a safer and much less invasive method to characterize RCC subtypes.

Previous studies have focused on the use of enhancement of lesions at multiphasic computed tomography (CT) or magnetic resonance imaging (MRI) to differentiate 
ccRCC from papillary RCC (pRCC) or chromophobe RCC (chRCC) $)^{9-12}$. But it's still challenging to make the right diagnosis of RCC subtype as the interpretation of imaging findings is a subjective process and an accurate prediction of histological RCC subtype strongly depends on the experience and expertise of radiologists. Besides, due to lack of validation on a larger scale, clinical application of these methods is limited. We need a more objective method to help us improve diagnosis of RCC subtype.

Texture analysis (TA) is a emerging imaging tool to quantify tissue heterogeneity that may not be perceived by the naked eye. It involves an objective computer-aided measurement of gray-level patterns within the lesions. A few studies have reported that TA could accurately differentiate renal fat-poor angiomyolipoma from $\mathrm{RCC}^{13-16}$. However, whether TA could discriminate RCC subtypes has been controversial. Raman et $\mathrm{al}^{16}$ and Yan $\mathrm{L}$ et $\mathrm{al}^{13}$ demonstrated that CT texture analysis (CTTA) could differentiate ccRCC from $\mathrm{pRCC}$, but Hodgdon et $\mathrm{al}^{14}$ showed there was no difference between ccRCC, pRCC and chRCC on unenhanced CT images. Further studies are needed to investigate the potential value of CTTA in characterizing RCC subtypes. As stated earlier that non-clear cell RCC (non-ccRCC) is not recommended for targeted 
therapies, discriminating non-ccRCC from ccRCC is far more clinically relevant, which could help patients with non-ccRCC avoid possible side effects and enormous cost of targeted agents. To our knowledge, the potential of CTTA has not been explored in this context of differentiating non-ccRCC from ccRCC.

The primary aim of this study was to retrospectively evaluate the diagnostic performance of CTTA to differentiate non-ccRCC from ccRCC and secondary aim was to investigate whether CTTA could differentiate non-ccRCC subtypes of pRCC and chRCC on multiphasic images.

\section{Materials and Methods}

\section{Patient Selection}

The institutional review board of our institution approved this retrospective study and waived the requirement for informed consent. Between June 2014 to August 2015, 165 patients with histologically confirmed diagnosis of RCC on entire tumor resection were identified. Twenty-nine patients were excluded for absence of preoperative multiphasic CT images in our picture archiving and communication system (PACS), including 24 patients with ccRCC, 3 patients with pRCC and 2 patients with chRCC. 
Two radiologists with 10 and 13 years of experience in abdominal imaging reviewed all the images and excluded another 10 patients with ccRCC in whom there was almost no solid component in the lesions for TA. The final study population comprised 126 patients and the details are provided in Fig 1.

\section{CT Examination}

All the patients underwent a preoperative three-phasic abdominal CT examination (Somatom Definition Flash; Siemens Healthcare, Forchheim, Germany). Protocol details are provided in Table 1. After the unenhanced scanning, intravenous injection of 100mL contrast material (Ultravist 370, Bayer ScheringPharma AG, Germany) followed by a $100-\mathrm{mL}$ saline chaser were administered at a rate of $4 \mathrm{~mL} / \mathrm{s}$. A bolustracking algorithm (CareBolus, Siemens Medical Solutions) was used and a region of interest (ROI) was placed in the thoracoabdominal aorta junction. The corticomedullary phase was initiated 5 seconds after the bolus-triggering threshold of $120 \mathrm{HU}$ was reached and the nephrographic phase occurred 50 seconds after the corticomedullary imaging acquisition. 


\section{CTTA}

All the images were de-identified before being accessed by the researchers. Two radiologists with 10 and 13 years of experience in abdominal imaging reviewed all the images and disagreement was resolved by consensus. Both reviewers were blinded to clinical and histological information. For each lesion in each phase, only one axial image with the optimal representation of the largest cross-sectional tumor area was chosen for TA. One of the two reviewers measured the maximal diameter of each lesion on the selected axial images and repeated the measurement several months later to assess the intra-observer agreement. Another radiologist also measured the maximal diameter of each lesion to assess the inter-observer agreement. All the selected images were exported from PACS and uploaded to the TexRAD commercial research software (TexRAD Ltd, www.texrad.com, part of Feedback Plc, Cambridge, UK). A single trained operator carried out all the TA and repeated the process eight weeks later. The average value of two measurements for each texture parameter was calculated and recorded.

For each lesion, a ROI was manually delineated slightly within the lesion border 
leaving an approximate distance of $1-2 \mathrm{~mm}$ from the outline in order to minimize volume averaging from surrounding perirenal fat or normal renal parenchyma. Confounding factors such as air, dense calcification and necrosis without enhancement were excluded when drawing the ROIs to minimize their impact on the CTTA (Fig 2A). Furthermore, the CTTA-algorithm setting excluded pixels with values less than -50 and greater than $200 \mathrm{HU}$ from inclusion in the texture analysis to increase the robustness in the quantification. Since the outline of lesions could be difficult to define on unenhanced images, the corresponding matched enhanced images of each lesion were referenced during delineation. ROIs for each axial section for matched unenhanced, corticomedullary and nephrographic CT images were then used for TA.

TA comprised a two-step process that involved selective scale image filtration to extract features of different sizes followed by texture quantification by histogram analysis. Image filtration utilized a Laplacian of Gaussian spatial band-pass filter to produce a series of derived images highlighting features at different spatial scales. The size of the image features highlighted by the filter is denoted by the Spatial Scaling Factor (SSF), which ranges between object radii of $0,2,3,4,5$ and $6 \mathrm{~mm}$. SSF can be 
considered as the width at which structures in the image will be highlighted while structures less than this width will become blurred. SSF 0 indicates used of no filtration (used as control), 2 indicates fine, 3-5 indicates medium and 6 indicates coarse texture scales respectively (Fig 2B-D). Six texture parameters were derived from histogram analysis: mean gray-level intensity (Mean, brightness), standard deviation (SD, degree of variation from the mean), entropy (irregularity of pixel intensity), mean of positive pixels (MPP), skewness (symmetry of the histogram distribution), and kurtosis (sharpness of the histogram distribution) ${ }^{17}$. For each lesion in each phase, all the six parameters across each SSF were recorded.

\section{Statistical Analysis}

Intra- and inter-observer agreement were calculated for the measurement of the maximum diameter of the lesions by using the intraclass correlation coefficient (ICC) test $(0.00-0.20$, poor agreement; $0.21-0.40$, fair agreement; $0.41-0.60$, moderate agreement; 0.61-0.80, good agreement; 0.81-1.00, excellent agreement). Papillary RCC and chRCC were classified together as non-ccRCC. Mann-Whitney $U$ tests were used to compare the value of each texture parameter in each phase for the research 
purposes (SPSS for Mac, version 20, SPSS Inc., Chicago, Ill). Although a two-sided $P<0.05$ indicated a significant difference, to control for type I errors arising from multiple comparisons, we adjusted the individual significance level as proposed by Cross and Chaffin ${ }^{17}: p^{*}=p \times[k-x+1]^{-1}$, where $p^{*}$ is the adjusted significance level for an individual test, $\mathrm{p}$ is the nominal significance level for the sequence of tests, $\mathrm{k}$ is the total number of the tests, and $\mathrm{x}$ is the number of significant tests. Receiver operating characteristic (ROC) curves and the area under the ROC curves (AUC) were calculated for each texture parameter in each phase and Pearson's correlation coefficient (r) between texture features at each SSF in each phase was evaluated so that only one of the features that showed a high correlation $(r>0.90)$ was selected to build the classifiers. As an AUC $\geqslant 0.8$ generally indicates a good test, we selected all the texture features with $\mathrm{AUC} \geqslant 0.8$ as predictors. In cases when all the $\mathrm{AUCs}<0.8$, only three texture parameters with the top three AUC values were selected as predictors as to minimize the likelihood of overfitting. Combinations of predictors were used to train multiple support vector machine (SVM) classifiers, and ROC curves were computed by the predicted values of regression of SVM for each SSF in each phase to determine the 
optimal combination. An SVM with a nonlinear radial basis function kernel was implemented in the 'SVM' function in R Package 'e1071' (Version 1.6-7, R Software), using the Library for Support Vector Machines (or LIBSVM) library ${ }^{19}$. The stability of classification was evaluated through 10 -fold cross-validation.

\section{Results}

General characteristics of the 126 patients with 127 RCC lesions (100 ccRCC, 12 pRCC, 15 chRCC) are summarized in Table 2. The mean time from preoperative CT evaluation to nephrectomy was 20 days for all RCC (range, 0-35 days). For the measurement of maximum diameter of the lesions, we found excellent intra-observer (intraclass correlation coefficient, $\mathrm{ICC}=0.95)$ and inter-observer agreement $(\mathrm{ICC}=0.91)$. The average size of all the RCC was $(4.9 \pm 2.6) \mathrm{cm}$. There was no significant difference in the average size of lesions among ccRCC (4.5 \pm 2.1$)$, pRCC (6.5 \pm 3.7$)$ and chRCC (6.1 \pm 3.2$)$ groups $(P>0.05)$.

\section{Differentiating non-ccRCC from ccRCC}

The median and interquartile range (IQR) associated with each texture parameter, 
the corresponding $P$ and the area under the receiver operating characteristic (ROC) curve (AUC) values for assessing the difference between non-ccRCC and ccRCC are provided in Supplementary 1. Compared to ccRCC, all the six texture parameters quantified from unenhanced images and skewness quantified from enhanced images didn't show any significant differences in non-ccRCC $(P>0.001$ after Cross and Chaffin correction). However, on enhanced images, kurtosis was significantly higher while Mean, SD, entropy and MPP were significantly lower at all the texture scales (SSF 06) in non-ccRCC $(P<0.001$ after Cross and Chaffin correction). As Mean and MPP quantified from SSF0 on both unenhanced and enhanced images, SD and entropy quantified from SSF2 on enhanced images, SD and MPP quantified from SSF2-5 on enhanced images all had a Pearson's correlation coefficient over 0.90 , only one of these correlated features at each SSF in each phase was selected to build the classifiers.

Regression models produced by combining the optimal discriminative texture features are summarized in Table 3. To identify non-ccRCC from ccRCC, all the models resulted in AUCs greater than 0.7. On unenhanced images, the model incorporating MPP, skewness and kurtosis at SSF0 produced the highest AUC of $0.82 \pm 0.05(P<0.001)$ 
with $78 \%$ sensitivity and $86 \%$ specificity (Fig 3A). On corticomedullary images, the highest AUC of $0.94 \pm 0.03(P<0.001)$ was reached at SSF6 by the model incorporating SD, entropy, MPP and kurtosis with 89\% sensitivity and 92\% specificity (Fig 3B). On nephrographic images, the model incorporating entropy, MPP and kurtosis at SSF0 yielded the highest AUC of $0.89 \pm 0.04(P<0.001)$ with $89 \%$ sensitivity and $83 \%$ specificity (Fig 3C). The 10-fold cross-validation support vector machine (SVM) accuracy arranged from $76 \%$ to $88 \%$ for each combination of texture features (Table 3 ).

\section{Differentiation between pRCC and chRCC}

The median and IQR associated with each texture parameter, the corresponding $P$ and AUC values for differentiating between pRCC and chRCC are summarized in Supplementary 2. Only Mean and MPP quantified from SSF0 on corticomedullary images were significantly different $(P=0.002)$ between the two subtypes. Mean and MPP were both significantly lower in pRCC (Mean: median pRCC, 48.2; IQR, 38.160.0; median chRCC,72.4; IQR, 54.1-101.5; P=0.02) (MPP: median pRCC, 49.6; IQR, 40.0-61.1; median chRCC, 74.6; IQR,58.3-101.6; $P=0.002)$. The mean AUC \pm standard error associated with Mean $(0.84 \pm 0.08)$ and MPP $(0.85 \pm 0.07)$ were greater than 0.8 . 
As Mean and MPP quantified from SSF0 on both unenhanced and enhanced images, SD and entropy quantified from SSF3-6 on unenhanced images, SD and MPP quantified from SSF2 on enhanced images and SSF3, 4 on corticomedullary images all had a Pearson's correlation coefficient over 0.90 , only one of these correlated features at each SSF in each phase was selected to build the classifiers.

The results of different regression models to differentiate between $\mathrm{pRCC}$ and chRCC are summarized in Table 4. On unenhanced images, all the AUCs were significantly greater than 0.7 and the model incorporating entropy, MPP and kurtosis quantified from SSF6 produced an AUC of $0.84 \pm 0.08(P=0.077)$ with $67 \%$ sensitivity and $100 \%$ specificity (Fig 4A). On corticomedullary images at SSF0, SD combined with MPP and Skewness yielded an AUC of $0.96 \pm 0.04$ with $87 \%$ sensitivity and $92 \%$ specificity (Fig 4B). On nephrographic images, the highest AUC of $0.93 \pm 0.05(P<0.001)$ with $93 \%$ sensitivity and $83 \%$ specificity (Fig 4C) was reached at SSF0 by incorporating MPP, skewness and kurtosis. The 10-fold cross-validation SVM accuracy arranged from $30 \%$ to $78 \%$ for each combination of texture features (Table 4 ). 


\section{Discussion}

Our study results show that texture parameters of SD, entropy and MPP seem to be strong predictors of non-ccRCC while MPP and skewness are helpful texture features to further differentiate between $\mathrm{pRCC}$ and chRCC. Our results indicate that TA may serve as a non-invasive method to identify non-ccRCC and it could potentially help those patients avoid unnecessary drug toxicity and financial burden by predicting histological RCC subtype.

Our results showed that non-ccRCC had significantly lower Mean, SD, entropy, MPP and higher kurtosis compared to ccRCC on enhanced CT images. Specifically, MPP quantified from the coarse texture scale (SSF6) on corticomedullary images seems to be a valuable texture feature, and the combination of SD, entropy, MPP and kurtosis at SSF6 on corticomedullary images is the strongest classifier for non-ccRCC.

Generally, our results were comparable to previous studies of renal, brain and lung neoplasms ${ }^{20-24}$. Greater heterogeneity is known to be likely related to higher histological grade of malignancy and poorer prognosis ${ }^{5-26}$. We can infer from this study that heterogeneity between RCC subtypes is likely linked to increasing number of 
objects or features highlighted in the filtration step and their mean brightness difference compared to the background of lesions resulting in higher Mean, SD, entropy, MPP and lower kurtosis in ccRCC. Skewness tends to be zero with increasing number of objects highlighted, which may be a general characteristic of RCC subtypes and hence did not demonstrate to be a useful discriminator. Our results were consistent with previous studies that higher entropy and lower degree of texture features related to uniformity are characteristics of $\mathrm{ccRCC}^{13-14}$. As ccRCC is generally more aggressive than nonccRCC, it worth investigating the relationship between these texture characteristics and the prognosis of RCC. As for MPP, it has been correlated negatively with angiogenesis in non-small cell lung cancer ${ }^{22}$. To our knowledge, we were the first to show that lower MPP on enhanced CT images may help to identify non-ccRCC. And MPP may be quite a good parameter in addition to kurtosis in vascular tumors as shown previously in treatment response studies of metastatic RCC ${ }^{27}$. Given that MPP only considers the average intensity of pixels greater than zero and non-ccRCC tends to be generally hypovascular, lower MPP in non-ccRCC appears to be consistent with hypo-vascular tumors exhibiting fewer bright areas. Furthermore, amongst non-ccRCC, pRCC tends to be 
even more hypo-vascular, which potentially explains the even lower value of MPP in pRCC compared to chRCC. We hypothesize that MPP on contrast-enhanced CT images may be positively correlated with the blood supply of RCC and MPP may be a better marker of treatment response compared to size, density and perfusion. When we compared our results to similar studies evaluating MR images for classifying RCC subtypes, Chandarana et $\mathrm{al}^{28}$ found that Kurtosis and skewness were significantly lower in ccRCC. We had similar findings about Kurtosis, but we didn't find any difference of skewness among RCC subtypes. This could possibly be attributed to the differences between imaging modalities and physical properties being captured. The role of skewness on $\mathrm{CT}$ in discriminating RCC subtypes may need further investigation.

In our study, we analyzed both unenhanced and contrast-enhanced CT images. On unenhanced CT images, our results were slightly different from the results of previous studies. Hodgdon et $a 1^{14}$ reported that there was no texture difference between RCC subtypes on unenhanced CT images. In our study, individual texture parameter also showed no significant difference between RCC subtypes but combinations of different texture parameters could be used to help identify non-ccRCC from ccRCC and 
differentiate between pRCC and chRCC. One possible explanation could be combinations of texture parameters may help to enhance the detection of subtle but potentially discriminating features that individual parameter may not be able to reflect. Although the effects of iodinated contrast material on TA are still unclear, differences in texture features between unenhanced and contrast-enhanced CT images were observed in previous studies ${ }^{21,29}$. More texture parameters on enhanced images showed significant differences between RCC subtypes and models integrating texture parameters quantified from enhanced images demonstrated higher diagnostic accuracies. Our findings seem to support that CTTA on enhanced images may provide more useful information, specifically corticomedullary images, to identify non-ccRCC from ccRCC.

In our study, before building the classifiers, Pearson's correlation coefficient between each feature was evaluated to make sure that the features selected to train SVM were not highly correlated. As an $\mathrm{AUC} \geq 0.8$ generally indicates a good test, we defined the optimal discriminative texture features as those with $\mathrm{AUC} \geq 0.8$ and they were combined to train SVM at each SSF in each phase. But there were some circumstances 
that none of the texture parameters yielded an $A U C \geq 0.8$. In such situations and to minimize the likelihood of overfitting, the optimal discriminative texture features were selected as texture parameters with the top three AUC values. However, there are also other strategies to select and define SVM classifiers ${ }^{14,30}$. In this study, most crossvalidation accuracies for identifying non-ccRCC from ccRCC were above $80 \%$ and some model resulted in $96 \%$ sensitivity and 92\% specificity. However, when differentiating between $\mathrm{pRCC}$ and chRCC, cross-validation SVM accuracies were low (30\% to $78 \%$ ). But yet there was a model incorporating SD, MPP and skewness quantified from SSF 0 on corticomedullary images produced an AUC of $0.96 \pm 0.04$ $(P<0.001)$ with $87 \%$ sensitivity and $92 \%$ specificity. We think the low cross-validation accuracy is likely related to the small sample size and it's possible that the increase in sample size would improve cross-validation accuracy. Larger cohorts of $\mathrm{pRCC}$ and chRCC are necessary to validate our results.

Our study had several limitations. First, the retrospective nature of our study may lead to overestimation of the diagnostic accuracy. Second, although the sample size of non-ccRCC was comparable to that of previous studies involving differentiation of 
RCC subtypes by using CTTA, the small sample size may contribute to the low crossvalidation accuracy. More data should be pooled together to confirm our results. Third, as we excluded $10 \mathrm{ccRCC}$ which had almost no solid component, it's possible that texture characteristics of ccRCC reported in this study may not be comprehensive enough and our results may not be generalized directly to those primarily cystic or necrotic lesions. Fourth, as the main purpose of this study was to investigate whether CTTA could be used to discriminate RCC subtypes, we haven't evaluated the correlation between texture parameters and the histological grading within each subtype. But we think that our study results add to clinical adoption of TA for evaluating RCC besides standard morphological assessment, which would give clinicians more confidence in making the optimal therapeutic choice for RCC patients.

In conclusion, our results show that CTTA could potentially be used to accurately differentiate non-ccRCC from ccRCC, and further differentiate between pRCC and chRCC. Prospective studies in larger cohorts of patients are required to further validate our study results before implementing into clinical practice. 


\section{References}

1. Ljungberg B, Campbell SC, Choi HY, Jacqmin D, Lee JE, Weikert S, Kiemeney

LA. The epidemiology of renal cell carcinoma. Eur Urol 2011; 20: 615-621.

2. Ljungberg B, Bensalah K, Canfield S, Dabestani S, Hofmann F, Hora M, et al. EAU guidelines on renal cell carcinoma: 2014 update. Eur Urol 2015; 67: 913-924.

3. Patard JJ, Leray E, Rioux-Leclercq N, Cindolo L, Ficarra V, Zisman A, et al. Prognostic value of histologic subtypes in renal cell carcinoma: a multicenter experience. J Clin Oncol 2015; 23: 2763-2771.

4. Capitanio U, Cloutier V, Zini L, Isbarn H, Jeldres C, Shariat SF, et al. A critical assessment of the prognostic value of clear cell, papillary and chromophobe histological subtypes in renal cell carcinoma: a population-based study. BJU Int 2009;103:14961500.

5. Hudes G, Carducci M, Tomczak P, Dutcher J, Figlin R, Kapoor A, et al. Temsirolimus, interferon alfa, or both for advanced renal-cell carcinoma. N Engl J Med 2007; 356: 2271-2281.

6. Negrier S, Gravis G, Perol D, Chevreau C, Delva R, Bay JO, et al. Temsirolimus 
and bevacizumab, or sunitinib, or interferon alfa and bevacizumab for patients with advanced renal cell carcinoma (TORAVA): a randomised phase 2 trial. Lancet Oncol 2011; 12: 673-680.

7. Procopio G1, Verzoni E, Bracarda S, Ricci S, Sacco C, Ridolfi L, et al. Italian Trials in Medical Oncology (ITMO) group. Overall survival for sorafenib plus interleukin-2 compared with sorafenib alone in metastatic renal cell carcinoma (mRCC): final results of the ROSORC trial. Ann Oncol 2013; 24: 2967-2971.

8. Volpe A, Finelli A, Gill I.S, Jewett MA, Martignoni G, Polascik TJ, et al. Rationale for percutaneous biopsy and histologic characterisation of renal tumours. Eur Urol 2012; 62: 491-504

9. Young JR, Margolis D, Sauk S, Pantuck AJ, Sayre J, Raman SS. Clear cell renal cell carcinoma: discrimination from other renal cell carcinoma subtypes and oncocytoma at multiphasic multidetector CT. Radiology 2013; 267: 444-453.

10. Kim SH, Kim CS, Kim MJ, Cho JY, Cho SH. Differentiation of Clear Cell Renal Cell Carcinoma From Other Subtypes and Fat-Poor Angiomyolipoma by Use of Quantitative Enhancement Measurement During Three-Phase MDCT. AJR Am J 
Roentgenol 2016; 206: W21-28.

11. Cornelis F, Tricaud E, Lasserre AS, Petitpierre F, Bernhard JC, Le Bras Y, et al. Multiparametric magnetic resonance imaging for the differentiation of low and high grade clear cell renal carcinoma. Eur Radiol 2015; 25: 24-31.

12. Wang H, Cheng L, Zhang X, Wang D, Guo A, Gao Y, Ye H. Renal cell carcinoma: diffusion-weighted MR imaging for subtype differentiation at 3.0 T. Radiology 2010; 257:135-143.

13. Yan L, Liu Z, Wang G, Huang Y, Liu Y, Yu Y, Liang C. Angiomyolipoma with minimal fat: differentiation from clear cell renal cell carcinoma and papillary renal cell carcinoma by texture analysis on CT images. Acad Radiol 2015; 22:1115-1121.

14. Hodgdon T, McInnes MD, Schieda N, Flood TA, Lamb L, Thornhill RE. Can Quantitative CT Texture Analysis be Used to Differentiate Fat-poor Renal Angiomyolipoma from Renal Cell Carcinoma on Unenhanced CT Images? Radiology 2015; 276: 787-796.

15. Kim TY, Choi HJ, Hwang HG, Choi HK. Three-dimensional texture analysis of renal cell carcinoma cell nuclei for computerized automatic grading. J Med Syst 2010; 
34: 709-716.

16. Raman SP, Chen Y, Schroeder JL, Huang P, Fishman EK. CT texture analysis of renal masses: pilot study using random forest classification for prediction of pathology. Acad Radiol 2014; 21:1587-1596.

17. Cross, EM, Chaffin, WW. Use of the binomial theorem in interpreting results of multiple tests of significance. Educational and Psychological Measurement 1982; 42: 25-34.

18. Miles KA, Ganeshan B, Hayball MP. CT texture analysis using the filtrationhistogram method: what do the measurements mean? Cancer Imaging 2013;13:400-406. 19. Chang C, Lin C. LIBSVM: a library for support vector machines. ACM Trans Intell Syst Technol 2011; 2: 1-27.

20. Goh V, Ganeshan B, Nathan P, Juttla JK, Vinayan A, Miles KA. Assessment of response to tyrosine kinase inhibitors in metastatic renal cell cancer: CT texture as a predictive biomarker. Radiology 2011; 261: 65-171.

21. Ganeshan B, Goh V, Mandeville HC, Ng QS, Hoskin PJ, Miles KA. Non-small cell lung cancer: histopathologic correlates for texture parameters at CT. Radiology 
2013; 266: 326-336.

22. Ganeshan B, Panayiotou E, Burnand K, Dizdarevic S, Miles K. Tumour heterogeneity in non-small cell lung carcinoma assessed by CT texture analysis: a potential marker of survival. Eur Radiol 2012; 22:796-802.

23. Andersen MB, Harders SW, Ganeshan B, Thygesen J, Torp Madsen HH, Rasmussen F. CT texture analysis can help differentiate between malignant and benign lymph nodes in the mediastinum in patients suspected for lung cancer. Acta Radiol 2016; 57: 669-676.

24. Li Z., Mao Y., Li H., Yu G., Wan H., Li B. Differentiating brain metastases from different pathological types of lung cancers using texture analysis of T1 postcontrast MR. Magn Reson Med 2016; 76: 1410-1419.

25. Smith AD, Gray MR, del Campo SM, Shlapak D, Ganeshan B, Zhang X, et al. Predicting Overall Survival in Patients With Metastatic Melanoma on Antiangiogenic Therapy and RECIST Stable Disease on Initial Posttherapy Images Using CT Texture Analysis. AJR Am J Roentgenol 2015; 205: W283-293.

26. De Cecco CN, Ganeshan B, Ciolina M, Rengo M, Meinel FG, Musio D, et al. 
Texture analysis as imaging biomarker of tumoral response to neoadjuvant chemoradiotherapy in rectal cancer patients studied with 3-T magnetic resonance. Invest Radiol 2015; 50: 239-245.

27. Tian F, Hayano K, Kambadakone AR, Sahani DV. Response assessment to neoadjuvant therapy in soft tissue sarcomas: using CT texture analysis in comparison to tumor size, density, and perfusion. Abdom Imaging 2015; 40:1705-1712.

28. Chandarana H, Rosenkrantz AB, Mussi TC, Kim S, Ahmad AA, Raj SD, et al. Histogram analysis of whole-lesion enhancement in differentiating clear cell from papillary subtype of renal cell cancer. Radiology 2012; 265: 790-798.

29. Ng F, Ganeshan B, Kozarski R, Miles KA, Goh V. Assessment of primary colorectal cancer heterogeneity by using whole-tumor texture analysis: contrastenhanced CT texture as a biomarker of 5-year survival. Radiology 2013; 266: 177-184. 30. Ng F, Kozarski R, Ganeshan B, Goh V. Assessment of tumor heterogeneity by CT texture analysis: can the largest cross-sectional area be used as an alternative to whole tumor analysis? Eur J Radiol 2013; 82: 342-348. 
Table 1. Scan parameters of CT examination

\begin{tabular}{ll}
\hline Parameter & \\
\hline Tube potential, kV & 120 \\
\hline Tube current, mAs & $\begin{array}{l}\text { Variable tube current with automatic tube- } \\
\text { current modulation activated }\end{array}$ \\
\hline Pitch & 0.9 \\
\hline Gantry rotation time, second & 0.28 \\
\hline Collimation, mm & $128 \times 0.6$ \\
\hline Kernel & B30f (medium smooth) \\
\hline Acquisition slice thickness, mm & 5 \\
\hline Field of view, mm & 300 \\
\hline
\end{tabular}


Table 2. Characteristics of patients and renal cell carcinomas.

\begin{tabular}{|c|c|c|c|c|c|}
\hline \multirow{2}{*}{ Characteristic } & \multirow{2}{*}{ All } & \multirow{2}{*}{$\mathrm{ccRCC}$} & \multicolumn{3}{|c|}{ non-ccRCC } \\
\hline & & & All & $\mathrm{pRCC}$ & $\operatorname{chRCC}$ \\
\hline \multicolumn{6}{|l|}{ Sex ${ }^{*}$} \\
\hline Male & $83(66)$ & $66(67)$ & $17(63)$ & $10(83)$ & $7(47)$ \\
\hline Female & $43(34)$ & $33(33)$ & $10(37)$ & $2(17)$ & $8(53)$ \\
\hline Age $(y)^{* *}$ & $54 \pm 12$ & $55 \pm 12$ & $51 \pm 15$ & $53 \pm 18$ & $49 \pm 13$ \\
\hline \multicolumn{6}{|l|}{ Side } \\
\hline Left & $66(52)$ & $55(55)$ & $11(41)$ & $5(42)$ & $6(40)$ \\
\hline Right & $61(48)$ & $45(45)$ & $16(59)$ & $7(58)$ & $9(60)$ \\
\hline
\end{tabular}

Data are numbers of lesions and data in parentheses are percentage, unless otherwise

indicated. *Data are numbers of patients. There was no significant difference in the portion of male patients among ccRCC, pRCC and chRCC groups $(P>0.05) .{ }^{* *}$ Data are mean \pm standard deviation. There was no significant difference in mean age among ccRCC, pRCC and chRCC groups $(P>0.05)$. Note: ccRCC $=$ clear cell RCC, non$\mathrm{ccRCC}=$ non-clear cell $\mathrm{RCC}, \mathrm{pRCC}=$ papillary $\mathrm{RCC}, \mathrm{chRCC}=$ chromophobe $\mathrm{RCC}$. 
Table 3. ROC Curves for Combinations of Texture Features for differentiating

non-ccRCC from ccRCC.

\begin{tabular}{|c|c|c|c|c|c|c|c|}
\hline Phase & $\mathrm{SSF}$ & Model & $\mathrm{AUC}^{*}$ & $P$ & $\mathrm{SVM}^{+}$ & Sen & Spe \\
\hline \multirow{6}{*}{ UE } & 0 & MPP + skewness + kurtosis ${ }^{* *}$ & $0.82 \pm 0.05$ & $<0.001$ & 79 & 0.78 & 0.86 \\
\hline & 2 & Mean + skewness + kurtosis & $0.79 \pm 0.06$ & $<0.001$ & 79 & 0.79 & 0.86 \\
\hline & 3 & Mean+ MPP+ kurtosis & $0.73 \pm 0.06$ & $<0.001$ & 80 & 0.59 & 0.85 \\
\hline & 4 & MPP + skewness + kurtosis & $0.80 \pm 0.06$ & $<0.001$ & 80 & 0.74 & 0.83 \\
\hline & 5 & entropy + MPP + kurtosis & $0.74 \pm 0.06$ & $<0.001$ & 76 & 0.59 & 0.84 \\
\hline & 6 & entropy+ MPP+ kurtosis & $0.76 \pm 0.06$ & $<0.001$ & 76 & 0.63 & 0.88 \\
\hline \multirow{6}{*}{$\mathrm{CM}$} & 0 & entropy + MPP + kurtosis & $0.92 \pm 0.04$ & $<0.001$ & 86 & 0.85 & 0.88 \\
\hline & 2 & Mean+ entropy+ MPP & $0.92 \pm 0.03$ & $<0.001$ & 87 & 0.89 & 0.90 \\
\hline & 3 & entropy+ MPP + kurtosis & $0.93 \pm 0.03$ & $<0.001$ & 88 & 0.96 & 0.85 \\
\hline & 4 & entropy $+\mathrm{MPP}+$ kurtosis & $0.92 \pm 0.03$ & $<0.001$ & 87 & 0.89 & 0.88 \\
\hline & 5 & entropy $+\mathrm{MPP}+$ kurtosis & $0.92 \pm 0.03$ & $<0.001$ & 88 & 0.93 & 0.89 \\
\hline & 6 & $\mathrm{SD}+$ entropy $+\mathrm{MPP}+$ kurtosis $^{* *}$ & $0.94 \pm 0.03$ & $<0.001$ & 87 & 0.89 & 0.92 \\
\hline \multirow{6}{*}{$\mathrm{N}$} & 0 & entropy+ MPP+ kurtosis ${ }^{* *}$ & $0.89 \pm 0.04$ & $<0.001$ & 85 & 0.89 & 0.83 \\
\hline & 2 & entropy+ MPP+ kurtosis & $0.84 \pm 0.05$ & $<0.001$ & 81 & 0.74 & 0.89 \\
\hline & 3 & entropy+ MPP+ kurtosis & $0.83 \pm 0.05$ & $<0.001$ & 83 & 0.74 & 0.90 \\
\hline & 4 & entropy $+\mathrm{MPP}+$ kurtosis & $0.83 \pm 0.05$ & $<0.001$ & 87 & 0.70 & 0.90 \\
\hline & 5 & Mean+ entropy+ MPP & $0.88 \pm 0.04$ & $<0.001$ & 82 & 0.93 & 0.78 \\
\hline & 6 & $\mathrm{SD}+$ entropy $+\mathrm{MPP}$ & $0.89 \pm 0.03$ & $<0.001$ & 84 & 0.89 & 0.81 \\
\hline
\end{tabular}

${ }^{*}$ Data are mean AUC \pm standard error. ${ }^{+}$Accuracy of the SVM classifier after 10 -fold cross-validation. ${ }^{* *}$ Model with the highest AUC in each phase. Note: AUC=area under the ROC curve, $\mathrm{SSF}=$ spatial scaling factor, $\mathrm{SVM}=$ support vector machine, Sen=sensitivity, Spe=specificity, Mean=mean gray-level intensity, $\mathrm{SD}=$ standard deviation, $\mathrm{MPP}=$ mean of positive pixels, $\mathrm{UE}=$ unenhanced, $\mathrm{CM}=$ corticomedullary, $\mathrm{N}=$ nephrographic. 
Table 4. ROC Curves for Combinations of Texture Features for differentiating

\section{between pRCC and ccRCC.}

\begin{tabular}{|c|c|c|c|c|c|c|c|}
\hline Phase & SSF & Model & $\mathrm{AUC}^{*}$ & $P$ & $\mathrm{SVM}^{+}$ & Sen & Spe \\
\hline \multirow{6}{*}{ UE } & 0 & entropy+ MPP + skewness & $0.76 \pm 0.10$ & 0.025 & 41 & 0.53 & 0.92 \\
\hline & 2 & SD + entropy + skewness & $0.83 \pm 0.08$ & 0.003 & 59 & 0.93 & 0.58 \\
\hline & 3 & entropy $+\mathrm{MPP}+$ kurtosis & $0.81 \pm 0.08$ & 0.006 & 41 & 1.00 & 0.50 \\
\hline & 4 & entropy + MPP + kurtosis & $0.79 \pm 0.09$ & 0.010 & 48 & 0.53 & 1.00 \\
\hline & 5 & entropy $+\mathrm{MPP}+$ kurtosis & $0.78 \pm 0.09$ & 0.013 & 52 & 0.60 & 0.92 \\
\hline & 6 & entropy+ MPP+ kurtosis ${ }^{* *}$ & $0.84 \pm 0.08$ & 0.077 & 52 & 0.67 & 1.00 \\
\hline \multirow{6}{*}{$\mathrm{CM}$} & 0 & $\mathrm{SD}+\mathrm{MPP}+$ skewness $^{* *}$ & $0.96 \pm 0.04$ & 0.000 & 78 & 0.87 & 0.92 \\
\hline & 2 & entropy + MPP + kurtosis & $0.77 \pm 0.10$ & 0.017 & 59 & 0.93 & 0.67 \\
\hline & 3 & entropy $+\mathrm{MPP}+$ kurtosis & $0.76 \pm 0.10$ & 0.025 & 44 & 0.87 & 0.58 \\
\hline & 4 & entropy $+\mathrm{MPP}+$ kurtosis & $0.80 \pm 0.09$ & 0.008 & 41 & 0.73 & 0.83 \\
\hline & 5 & $\mathrm{SD}+$ entropy+ MPP & $0.64 \pm 0.11$ & 0.223 & 30 & 0.73 & 0.67 \\
\hline & 6 & $\mathrm{SD}+$ entropy $+\mathrm{MPP}$ & $0.68 \pm 0.11$ & 0.118 & 37 & 0.53 & 0.92 \\
\hline \multirow{6}{*}{$\mathrm{N}$} & 0 & MPP + skewness + kurtosis $^{* *}$ & $0.93 \pm 0.05$ & 0.000 & 70 & 0.93 & 0.83 \\
\hline & 2 & entropy $+\mathrm{MPP}+$ skewness & $0.74 \pm 0.10$ & 0.032 & 37 & 0.93 & 0.50 \\
\hline & 3 & entropy $+\mathrm{MPP}+$ skewness & $0.78 \pm 0.09$ & 0.013 & 44 & 0.60 & 1.00 \\
\hline & 4 & MPP+ skewness + kurtosis & $0.84 \pm 0.08$ & 0.002 & 41 & 0.67 & 1.00 \\
\hline & 5 & Mean + SD + kurtosis & $0.88 \pm 0.07$ & 0.001 & 41 & 0.67 & 1.00 \\
\hline & 6 & $\mathrm{SD}+$ entropy + kurtosis & $0.91 \pm 0.06$ & $<0.001$ & 63 & 0.87 & 0.92 \\
\hline
\end{tabular}

${ }^{*}$ Data are mean AUC \pm standard error. ${ }^{+}$Accuracy of the SVM classifier after 10 -fold cross-validation. ${ }^{* *}$ Model with the highest AUC in each phase. Note: AUC=area under the ROC curve, $\mathrm{SSF}=$ spatial scaling factor, $\mathrm{SVM}=$ support vector machine, Sen=sensitivity, Spe=specificity, Mean=mean gray-level intensity, $\mathrm{SD}=$ standard deviation, $\mathrm{MPP}=$ mean of positive pixels, $\mathrm{UE}=$ unenhanced, $\mathrm{CM}=$ corticomedullary, $\mathrm{N}=$ nephrographic. 
Fig 1. Flowchart of patient population and exclusion criteria.

Note: $\mathrm{ccRCC}=$ clear cell $\mathrm{RCC}$, non-ccRCC=non-clear cell $\mathrm{RCC}, \mathrm{pRCC}=$ papillary $\mathrm{RCC}$, chRCC $=$ chromophobe $\mathrm{RCC}$.

Fig 2. Demonstration of ROI delineation within the lesion and corresponding texture images of different texture scales.

Corticomedullary CT image in a patient with right renal lesion shows region of interest (blue contour) drawn within lesion border (A). Corresponding texture analysis images display fine (B), medium (C) and coarse (D) textures obtained by using SSF 2,4,6 respectively.

Fig 3. ROC curves for SVM performances with the highest AUC value on (A) unenhanced, (B) corticomedullary and (C) nephrographic phase images to identify non-ccRCC from ccRCC.

Fig 4. ROC curves for SVM performances with the highest AUC value for differentiating between pRCC and chRCC on (A) unenhanced, (B) corticomedullary and (C) nephrographic phase images. 


\section{Supporting Information}

Supplementary 1. The median, inter-quartile range, $P$ value and $A U C$ value for

each texture parameter for discriminating non-ccRCC from ccRCC on multiphasic CT images.

Supplementary 2. The median, inter-quartile range, $P$ value and AUC value for each texture parameter for differentiating between $\mathrm{pRCC}$ and chRCC on multiphasic CT images.

Note: $\mathrm{SSF}=$ spatial scaling factor, Mean=mean gray-level intensity, $\mathrm{SD}=$ standard deviation, $\mathrm{MPP}=$ mean of positive pixels, $\mathrm{UE}=$ unenhanced, $\mathrm{CM}=$ corticomedullary, $\mathrm{N}=$ nephrographic, $\mathrm{pRCC}=$ papillary renal cell carcinoma, non-chRCC $=$ chromophobe renal cell carcinoma, $\mathrm{AUC}=$ area under the $\mathrm{ROC}$ curve. 